\title{
Mass-loss predictions and stellar masses of early-type stars
}

\author{
Alex de Koter \\ Sterrenkundig Instituut Anton Pannekoek, Universiteit van Amsterdam, \\ Kruislaan 403, NL-1098SJ Amsterdam, Nederland \\ Jorick S. Vink \\ Imperial College, Blackett Laboratory, \\ Prince Consort Road, London, SW72BZ, UK
}

\begin{abstract}
We show that the stellar masses implied by our predictions of the wind properties of massive stars are in agreement with masses derived from evolution theory and from direct measurements using spectroscopic binaries, contrary to previous attempts to derive masses from wind theory.
\end{abstract}

\section{Introduction}

The stellar winds of early-type stars are thought to be driven by radiation pressure on spectral lines. Predictions of wind properties are usually tested by comparing them to $\dot{M}$ and $v_{\infty}$ values derived for a set of about 30 well studied Galactic and Magellanic Cloud O- and early B-type stars (Puls et al. 1996). Although it has proven challenging enough to match predictions with observations, these stars provide only a limited test. To better constrain wind theory, one should confront a wider range of predictions with observations. Meaningful new tests include extending the comparisons of $\dot{M}$ and $v_{\infty}$ to: $(i)$ extremely luminous Of and WN5-6h stars; (ii) stars at (both sides of) the bi-stability jump, an abrupt discontinuity in $v_{\infty}$ found to occur at spectral type $\mathrm{B} 1 ;$, and (iii) Luminous Blue Variables. We have applied our predictions of mass loss to all the above cases with excellent results (de Koter et al. 1997; Vink et al. 1999, 2000,2002 ).

As the properties of stellar winds depend also on stellar mass, an alternative test would be to compare masses derived from line-driven wind theory with those of independent methods. This is a relevant issue in view of current problems with masses based on mass-loss rates and terminal wind velocities.

\section{Stellar masses derived from wind theory}

Although some wind properties (of O-type stars) are almost mass-independent, such as the modified wind momentum $\dot{M} v_{\infty} \sqrt{R}$, the mass loss itself behaves as $\dot{M} \propto M_{*}^{-1.3}$ (Vink et al. 2000). So, in principle, the mass may be derived when all relevant stellar properties - including $\dot{M}$ and $v_{\infty}$ - are known. So far, attempts to derive $M$ from wind theory yielded systematically lower masses (up to tens of percent) than those derived from evolutionairy tracks, but appeared 


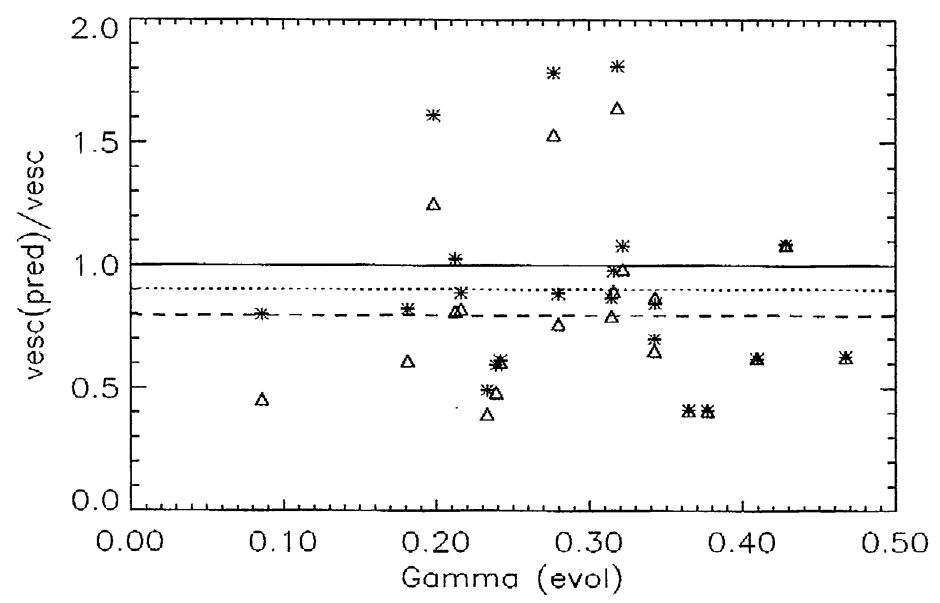

Figure 1. Consistency check for O-type stars between predicted surface $v_{\text {esc }}$ and $v_{\text {esc }}$ implied by the stellar parameters. Symbols explained in text.

to be in agreement with spectroscopic mass determinations (e.g., Herrero et al. 2001).

Using the latest generation of model atmospheres (see Herrero in these Proceedings), the spectroscopic masses now appear to be in much better agreement with $M_{\text {evol }}$. Also, direct mass determinations using spectroscopic binaries (e.g., Massey et al. 2002) seem to favor evolutionairy masses. This would leave the wind masses as the exception. However, here we present evidence that also our mass-loss predictions favor $M_{\text {evol }}$. Figure 1 shows a consistency check between predicted surface escape velocity $v_{\mathrm{esc}} \propto\left[M_{*}(1-\Gamma)\right]^{1 / 2}$ and $v_{\text {esc }}$ implied by the stellar parameters. $\Gamma$ is the Eddington factor for electron scattering. The prediction involves the observed mass loss and terminal wind velocity, i.e., $v_{\text {esc }}$ (pred) $=f\left(\dot{M}, v_{\infty}, L_{*}, T_{\text {eff }}, M_{*}\right)$. The case for the evolutionary masses is shown using asterisk symbols; 'old' spectroscopic masses (in agreement with previous attempts to derive masses from wind theory) have triangle symbols. The mean ratio for $M_{\text {evol }}$ (dotted line) is $0.90 \pm 0.16$; for $M_{\text {spec }}$ (dashed line) it is $0.79 \pm 0.12$.

This shows that within the error our predicted wind properties are in agreement with evolutionairy masses, bringing also the masses from wind theory in accord with those derived from all other mass determination methods.

\section{References}

de Koter, A., Heap, S.R., Hubeny, I. 1997, ApJ 477, 792

Herrero, A., Puls, J., Corral, J., Kudritzki, R.-P., Villamariz, M.R. 2001, A\&A 366, 623

Massey, P., Penny, L.R., Vukovich, J. 2002, ApJ 565, 982

Puls, J., Kudritzki, R.-P., Herrero, A. 1996, A\&A 305, 171

Vink, J.S., de Koter, A., Lamers, H. 1999, A\&A 350, 181

Vink, J.S., de Koter, A., Lamers, H. 2000, A\&A 362, 295

Vink, J.S., de Koter, A. 2002, A\&A 393, 543 